\title{
A Low-cost Capacitive Fuel-level and Quality Sensor for Automotive Applications
}

\author{
Dr.-Ing. Volker Skwarek ${ }^{1}$, Dipl.-Ing. Torsten Eggers ${ }^{1}$ \\ ${ }^{1}$ Hella Fahrzeugkomponenten GmbH, Dortmunder Str. 5, 28199 Bremen, Germany \\ volker.skwarek@hella.com
}

\begin{abstract}
Due to internationally significant differences in fuel ingredients and the resulting fuel quality, modern direct injection gasoline and diesel engines need to be able to adapt their point of optimal operation according to the used type off fuel. This is necessary to achieve optimum fuel efficiency, exhaust gas minimization and protection of exhaust gas after-treatment components. Therefore it is important to understand at least minimal qualitative properties of the fuel in the car's tank. In future fuel scenarios and emissions regulation standards this is no longer possible with software based virtual sensors based on lambda and knock-sensor read-out only, because the control is delayed.
\end{abstract}

The conventional fuel level sensors based on float and potentiometers - nowadays encapsulated only deliver fuel level information at relatively high cost. They cannot provide fuel quality, temperature and mixture information and feature a passive (resistive) output only. In most saddle fuel tank configurations two are used to get accurate fuel level information to estimate remaining mileage.

With a capacitive sensor based on a specific printed circuit board design it is possible to measure five characteristic properties of fuel level and quality with only one sensor structure: fuel level, major components, fuel layering by decomposition, tank inclination and fuel movement.

Basic principles of the measurement system proposed are a combination of capacitance measurement and impedance spectroscopy. In this paper the concept of this new sensor and first results of simulation and measurements are presented.

Key words: level sensor, fuel, capacitive, fuel quality, impedance spectroscopy

\section{Motivation}

Automotive fuel consistency $(=$ normative purity) varies internationally significantly. Furthermore the higher the potential shortage of oil the more alternative fuel ingredients such as ethanol are added. Unfortunately as well the optimum point of operation of the engine has to be adapted to the fuel as parts with permanent contact with potentially aggressive fuel may be damaged.

One of these parts to be protected is the currently most popularly used fuel level sensing principle: Floats in the fuel "swimming" on the surface. With a usually metal rod connected to a potentiometer its resistance is a measure for the fuel level. But some fuel components increase corrosion of the metal whereas more precious - and more expensive - materials have to be used, making this sensing principle more expensive. Because there is no additional functionality within the conventional sensing principle the price to performance ratio becomes unbalanced. This is even worse in up- to date fuel tank designs, because in most cases two fuel level sensor, in rare cases like four-well drive cars even four sensors, have to be used due to the saddle-like geometry.

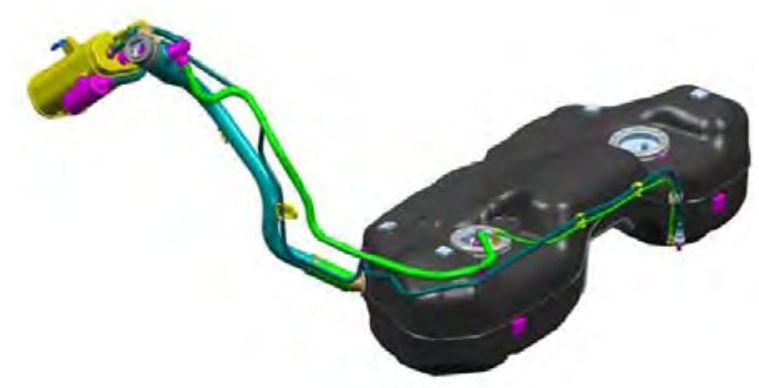

Fig. 1 Complete fuel tank (saddle configuration) with two fuel supply modules [Source: Delphi].

In each of the two separate volumes in Fig. 1 one fuel supply module is mounted, both of them equipped with a float based fuel level sensor. 


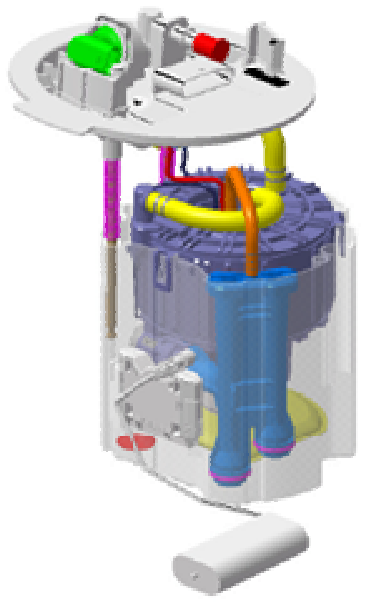

Fig. 2 Fuel supply module with fuel pump etc. and fuel level sensor (in front) [Source: Delphi].

Several new challenges have to be solved with an innovative fuel level sensor in order to rebalance the price to performance (functionality) ratio:

- Using no metal in contact with fuel

- Using no moving parts

- Determining at least basic fuel components such as conventional fuel, water, ethanol content of gasoline fuel, biodiesel content of diesel fuel

- Optionally, detect quality relevant fuel properties like e.g. evaporation behavior without using a complex method like e.g. near infrared absorption spectroscopy used in dedicated in-line fuel quality sensors currently under development [1,2]

- Introduce diagnostic features, power-down and digital bus interface

\section{Capacitive fuel measurement}

Although capacitive level sensing is a wellknown principle $[3,4,5]$, it has never established in automotive fuel level measurement so far, probably because of its sensitivity against side effects like changes in dielectric constant and conductivity of the fluid. But with increasing processor power even within sensors and an intelligent capacitive structure the compensation of the side effects is possible with the advantage of measuring more physical properties but only level.

Precisely, the new approach is able to measure respectively detect the following properties:

- Fuel level

- Fuel composition, layering

- Inclination

- $\quad$ Sloshing

\section{Capacitive sensor element}

The capacitive sensor element consists principally of etched stacked capacitors on a standard printed circuit board for determining the fuel level itself. As the fuel consistency does not need to be homogenous in the tank it might be complicated to determine an exact level with a deviation of less than $2 \%$ within a potential mixture of different fuel components. For that reason reference elements are integrated into the capacitive structures to obtain the local values for the dielectric constant [see Tab. 1] used for compensation of neighbored level measuring stacks.

Tab. 1: Dielectric constant of media in fuel system (for measurements at low field strength, at room temperature with e.g. $100 \mathrm{kHz}$ to $1 \mathrm{MHz}$ frequency)

\begin{tabular}{|c|c|}
\hline Media & $\varepsilon_{\mathrm{r}}[-]$ \\
\hline Gasoline & 2.2 \\
\hline Diesel & 2.2 \\
\hline Ethanol & $\sim 24$ \\
\hline Methanol & $\sim 30$ \\
\hline Water & $\sim 80$ \\
\hline Air & 1.0034 \\
\hline
\end{tabular}

Furthermore, with a specially designed differential sub structure of these capacitive stacks the inclination of the fuel can be calculated by differential capacitive measurement. And a fast changing inclination is a sign of fluid sloshing; therefore the measurements can be integrated over a period of the movement.
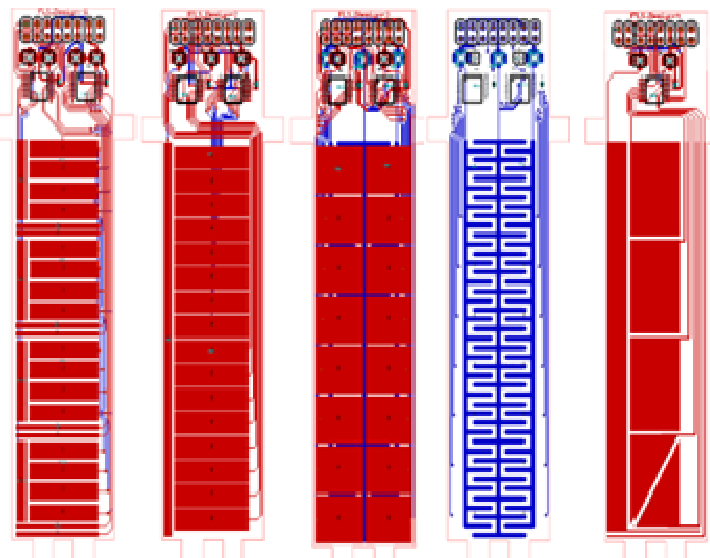

Fig. 3 Printed circuit board test layout structures; left to right: level with intermediate references (1), level calculation by center of gravity calculation (2), simple level (3), inclination (4), simplified from (1) (5)

To obtain the second most important information of this sensor, the potential fluid 
components, the $\varepsilon_{\mathrm{r}}$ between different capacitive electrodes in the capacitor stack will be measured. With significantly different $\varepsilon_{\mathrm{r}}$ for water, regular fuel, air and alcohols (see Tab. 1) these ingredients can easily be detected. Furthermore, utilizing the frequency dependency of $\varepsilon_{\mathrm{r}}$ and tan $\delta$, these components can even be sub-divided by performing a frequency sweep and measuring the impedance at different frequency points. With this electrical spectroscopy method the fluid can be basically examined for different components.

\section{Sensor design}

The complete electrical equivalent network of the impedance measurement sensor arrangement and the surrounding fluid are depicted in Fig. 4.

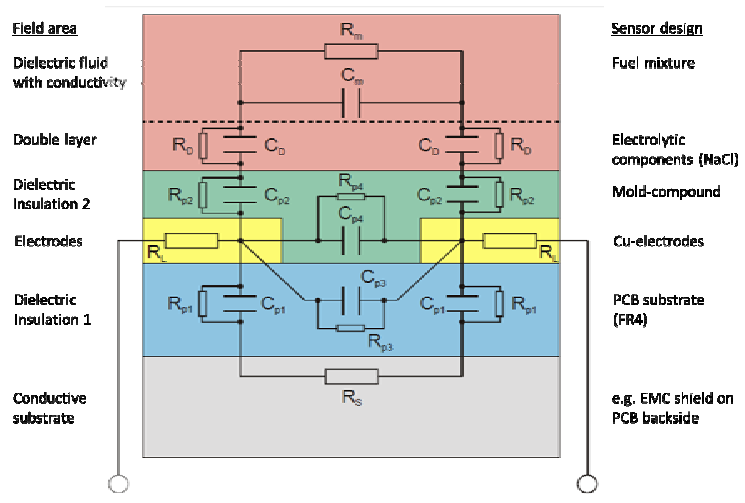

Fig. 4 Electrical equivalent network of sensor arrangement and electrolytic fluid to be analyzed

The network can be simplified taking into account, that the dielectric insulation 1 (PCB substrate FR4), the dielectric insulation 2 (molded epoxy polymer) can both be assumed as ideally non-conducting at low measurement frequencies, thus $R_{p 2}=\infty$. The conductivity of the backside shielding layer (Cu-plane) can be set as infinite, thus $R_{s}=0$.

The electrochemical double-layer capacitance is neglected (shortcut) in this application, because of measurement frequencies $>1 \mathrm{KHz}$ compared to the very high capacitance due to $\mathrm{nm}$ range typical double layer thickness.

The pure fuel always shows a specified minimum conductivity to comply with electrostatic discharge protection requirements. On the other hand, fuel conductivity might be up to $10 \mu \mathrm{S} / \mathrm{cm}$ due to the penetration of impurities during fuel refilling. Therefore, the fuel conductivity cannot be neglected. Additionally, especially ethanol is hygroscopic: E100 (100\% ethanol fuel) can take-up up to $7 \%$ water from the environment. The conductivity of gasoline, ethanol mixtures has been studied in detail [6].
Taking into account all assumptions, the electrical equivalent network can be simplified as depicted in Fig.

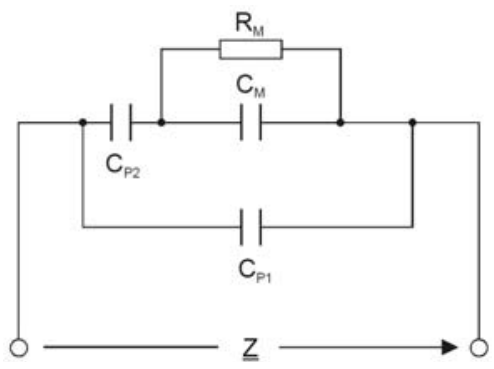

Fig. 5 Simplified electrical equivalent network of sensor arrangement and electrolytic fluid to be analyzed

The resulting real-world fuel mixture situation is more complex than binary mixtures of two pure fluids. Therefore, an effective calculation of $\varepsilon_{r}$ based on a physical modeling approach like e.g. Debye-models is hardly possible.

\section{Sensor manufacturing}

The sensor printed circuit board is populated with all SMDs and afterwards over-molded with a thin layer of epoxy polymer (EP) by injection molding to ensure long-term stability of the electronics and capacitor stack against chemically aggressive fuels. In a second molding step, a connector and fixation structures are built-up from fuel resistant polyamide thermoplastics (see Fig. 6).

A flexible tooling concept is applied to cope with different lengths of the sensor for different tank geometries. The maximum measurement range is $300 \mathrm{~mm}$. Different connectors as well as fixations can be used in the second molding step by individual tools.

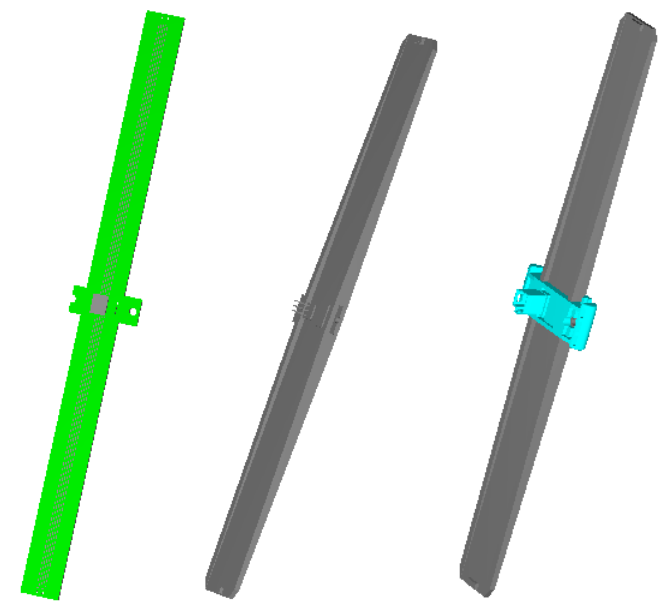

Fig. 6 Sensor manufacturing process steps: 1. Sensor PCB with press-fit pins and all $S M D$ populated (left); 2. Sensor PCB over-molded with thermoset EP material with stiffening structure (middle); 3. Completed sensor with molded thermoplastics connector/ fixation element (right) 


\section{Sensor modeling \& simulation}

A multi-physics FE-model has been set-up to optimize the sensor design and to understand and minimize the sensitivity to manufacturing process and material tolerances.

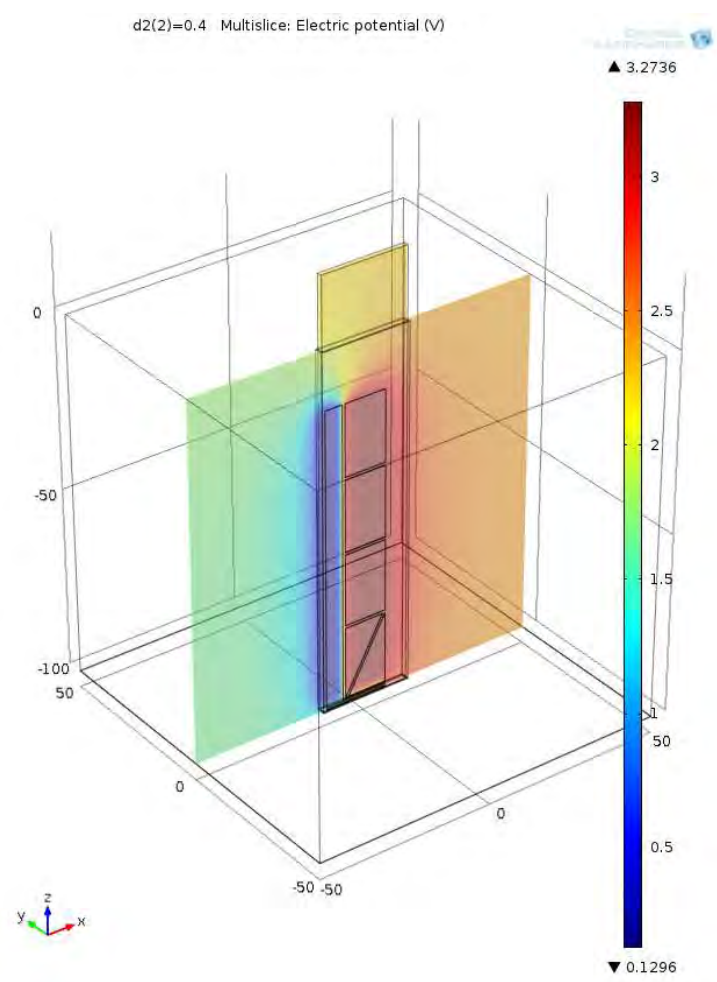

Fig. 7 Complete 3D FE-model to simulate electrical field distribution and extract capacitance values.

Utilizing the FE-simulation model (Fig. 7) the capacitive sensor structure design is optimized and manufacturing parameter tolerances are studied (Fig. 8). The capacitances of each stack element are extracted vs. fluid level for different fluids (see Fig. 9 and Fig. 10)

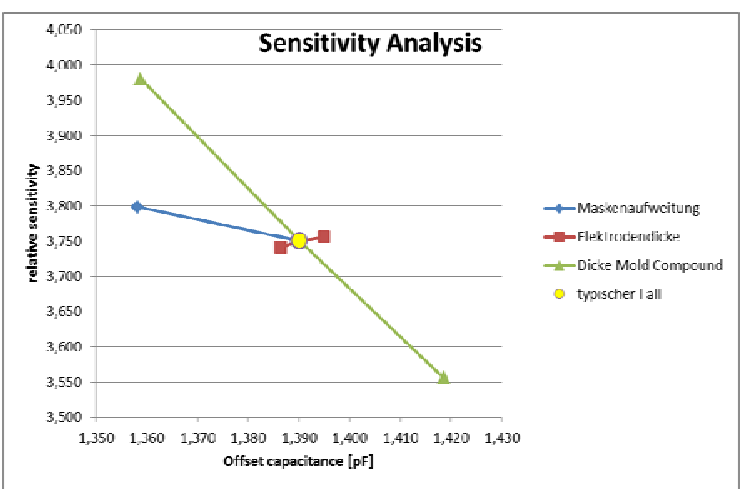

Fig. 8 Sensitivity analysis $\left(\varepsilon_{r}=24\right)$ for track width widening (blue), PCB Cu-electrode thickness variation (red), thickness variation of mold compound (green). The target design geometry is shown in yellow.

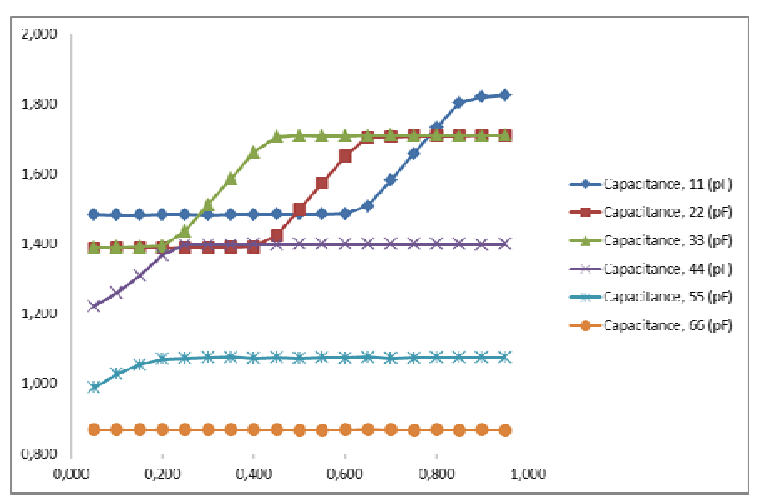

Fig. 9 Capacitance over normalized level for gasoline fuel $\left(\varepsilon_{r}=2.2\right)$ for the layout \#5 (see Fig. 3)

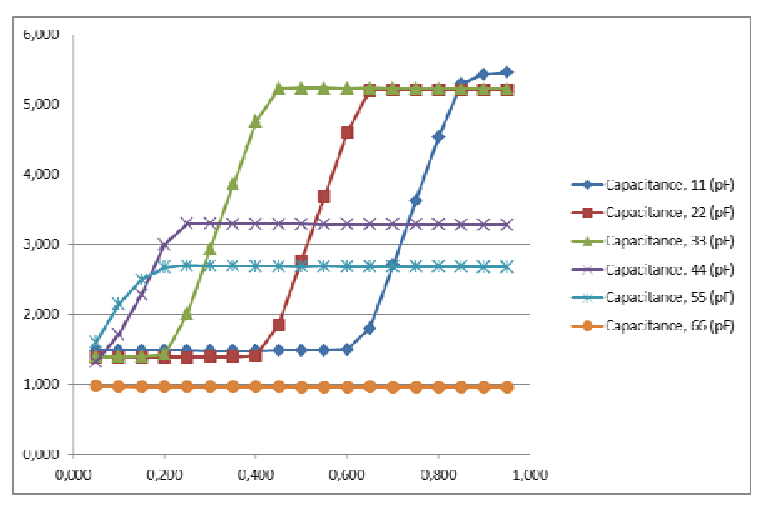

Fig. 10 Capacitance over normalized level for ethanol fuel $\left(\varepsilon_{r}=24.3\right)$ for the layout \#5 (see Fig. 3)

\section{Sensor Hardware, Software and Calibration Strategy}

For the level and mixture measurement the capacitances are multiplexed by analog multiplexers and converted by a single-channel capacitance to digital (CDC) application specific integrated circuit to a digital ${ }^{2} \mathrm{C}$ output. The selection of the capacitances to be converted is done by a small $8 / 16$ bit microcontroller also performing the necessary algorithms and calculations to compensate all influences, e.g. temperature dependency. In the lab set-up a mixed-signal programmable system-on-a-chip (PSOC) is used for the optional fuel quality measurement by impedance spectroscopy.

The end off line (EOL) in the automated manufacturing line will use a measurement at air with defined temperature and humidity and a fluid dummy measurement. By this method tolerances introduced by the production process, tool wear and material (e.g. FR4, mold compound) can be compensated. 


\section{Sensor Measurements}

Initial measurements could be performed up to now only because of some instability in early stage lab measurement set-up respectively prototype electronics configuration.

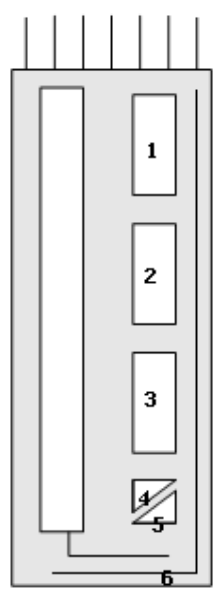

Fig. 11 Capacitances of basic measurement test structure for calculation of center of gravity from all mutual capacitances $C_{n}$ with $n=[1 . .5] . \quad C_{6}$ is the reference electrode. All capacitances are measured between numbered electrode and unnumbered counter electrode.

The lab prototype printed circuit boards only contain analog multiplexers with digital SPI control to switch the mutual capacitance electrodes to coaxial cable outputs connected to a LCR-meter or impedance analyzer or the respective measurement IC (single channel CDC or complex impedance measurement IC).

Tab. 2: Capacitances measured with Agilent LCR meter $4285 \mathrm{~A}\left(f_{M}=150 \mathrm{kHz}, U_{M}=500 \mathrm{mV} V_{p p}, D C_{B I A S}=\right.$ OV, open compensation, $2 m$ cable), sensor $P C B$ completely covered by fluid inside glass container

\begin{tabular}{|c|c|c|c|}
\hline $\begin{array}{c}\text { Capacitance } \\
{[\mathrm{pF}]}\end{array}$ & Air & Fuel & Ethanol \\
\hline $\mathrm{C}_{1}$ & 1,546 & 1.972 & 10.782 \\
\hline $\mathrm{C}_{2}$ & 1.548 & 1.983 & 11.710 \\
\hline $\mathrm{C}_{3}$ & 1.537 & 1.975 & 12,188 \\
\hline $\mathrm{C}_{4}$ & 1.430 & 1.815 & 10,404 \\
\hline $\mathrm{C}_{5}$ & 1.152 & 1.478 & 9,102 \\
\hline $\mathrm{C}_{6}$ & 1.199 & 1.474 & 7,900 \\
\hline
\end{tabular}

The sensor capacitances $\mathrm{C}_{1}-\mathrm{C}_{6}$ do include an additional offset, because there is neither perfect shielding nor guarding of the conductors leading to the measurement electrodes in this simple measurement set-up. Due to the missing guarding between the single capacitances, neighboring capacitances add additional stray capacitance.

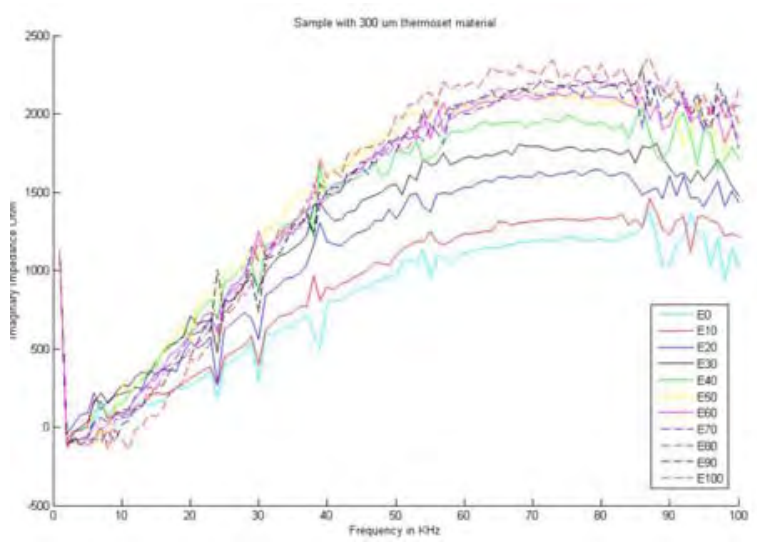

Fig. 12 Impedance spectra (imaginary part) for different ethanol concentrations $(0.3 \mathrm{~mm}$ mold thickness).

Using one of the fuel level capacitor arrangements for impedance spectroscopy one obtains curves like the one in Fig. 12. The ethanol concentration can be calculated by e.g. using a support vector machine algorithm.

\section{Summary and Outlook}

The first measurements show deviations between theoretic calculations, lab measurements and initial FE based simulations. The layout of the sensor PCB must be optimized.

Parallel to layout optimization a DoE will be setup to perform lab measurements for algorithm development and calibration strategy refinement.

The product idea and production concept enable the realization of this automotive sensor at competitive pricing level, which was not possible in the past. Next step in the development is the release of feasibility samples for initial design validation (vibration, thermal shock etc.) and first customer sampling.

\section{References}

[1] A. Lunati, J. Fournel, Innovative on board optical sensor dedicated to measure water, alcohols and ethers content in biofuels for Flexfuel engine optimization, SAE conference paper, 2008-012451 (2008)

[2] Euripides-Eureka research project IQfuel, http://www.euripideseureka.eu/img/projects/document/Press\%20Rele ase \%20IQFUEL\%20CONTINENTAL\%20EURIPI DES.pdf

[3] Patent application DE19916979A1, method for level measurement and level sensor, 1999 
[4] Patents US 6,502,460B1, Fluid level measuring system, Jan. 7, 2003; US 6,497,144B1 \& $6,498,566 \mathrm{~B}$, Method for measuring fluid level, Dec. 24, 2002, US 6,823,731B1, Liquid level sensing assembly and method for measuring using same, Nov. 30, 2004

[5] H. Steins et al., Selbstkompensierender kapazitiver Flüssigkeitenfüllstandssensor,
Sensoren im Automobil, 79-91(2006), ISBN-13: 978-3-8169-2648-1

[6] D.W. Kirk, Conductivity of gasoline-ethanol-water mixtures, FUEL, vol.62, December, 1512-1513 (1983) 